\title{
Thermal conductivity of tubular nanowire composites based on a thermodynamical
} model

\author{
G. Lebon and H. Machrafi*
}

Thermodynamics of Irreversible Processes, Liège university, 17 Allée du 6 Août, 4000 Liège, Belgium

*Corresponding author

Electronic mails: g.lebon@ulg.ac.be (G.Lebon), h.machrafi@ulg.ac.be (H. Machrafi)

\begin{abstract}
A formula for the effective heat conductivity of a nanocomposite with cylindrical nanowire inclusions is derived. Both transversal and longitudinal heating along the wires are investigated. Several effects are examined: the volume fraction and sizes of the nanowires, the type of scattering at the particle-matrix interface and temperature. As illustration, silicon nanowires inclusions in a germanium matrix is considered; the results are shown to be in good agreement with other models and numerical solutions of the Boltzmann transport equation. Our main contribution consists of using extended irreversible thermodynamics to cope with the nano dimensions of the wires.
\end{abstract}

Keywords: Thermal conductivity; Nanowire composites; Extended thermodynamics; Temperature-dependent phonon properties 


\section{Introduction.}

Thermal effects in nanomaterials are the subject of a huge amount of research both from theoretical and technological viewpoints. A question of particular and acute interest is the determination of the effective heat conductivity in nanocomposites. To apprehend the problems, several ways are open: either to solve directly the Boltzmann transport equation [1], to construct phenomenological models [2], to use atomistic simulations [3] or to mix both macroscopic and microscopic considerations which is the route followed in the present approach. More specifically, we consider two-dimensional tubular silicon nanowires embedded in a homogeneous germanium matrix; both cases with the heat flux directed normally and parallel to the axis of the Si wires are investigated To model heat transport in composites, we use a formalism, called the effective medium approximation (EMA), initiated by Nan et al [2] and revisited by Minnich and Chen [4]. Since the dimensions of the nano materials are comparable to the mean free path of the heat carriers, heat transport is mainly of ballistic rather than diffusive nature. Therefore, heat transport can no longer be described by Fourier's law and classical thermodynamics but requires a more sophisticated approach. Here, we will follow the line of thought of extended irreversible thermodynamics (EIT) which is especially suited to treat problems at sub-scales. The main idea underlying EIT $[5,6,7]$ is to upgrade the thermodynamic fluxes, like the heat flux to the rank of independent variable at the same level as the classical variables, as the temperature. The consequence is that Fourier's law will be substituted by more complex expressions allowing to deal with high frequency and small-scale systems. EIT has been applied in a previous paper [8] wherein we have studied heat transport in composites formed by spherical particles distributed randomly in a homogeneous matrix. The results were shown to be in good agreement with Monte Carlo simulations and other models $[9,10]$ using different schemes. In the present work, we explore 
further the applicability and limitations of the model developed in [8] by considering wires instead of spheres because the former play a critical role in a great number of technological applications.

The present paper is organized as follows. The model is set up in section 2. It consists in a mixing of EMA and EIT, whose main ingredients are briefly recalled. In Section 3, the model is applied to the problem of dispersion of Si nanowires in a Ge matrix: influence of volume fractions, size of the nano wires and the thermal boundary resistance are discussed. Temperature dependence of the overall heat conductivity, a topic ignored in most papers, is treated in Section 4. Conclusions are drawn in Section 5.

\section{The thermal conductivity model}

The basic relation is Maxwell's one [11] improved by Hasselman and Johnson [12] to include thermal boundary resistance and by Nan et al [2] who considered various particle shapes. Accordingly, the overall thermal conductivity of the composite in the direction perpendicular and normal to the heat flux are respectively given by

$$
\begin{aligned}
& \lambda_{\perp}=\lambda_{m} \frac{(1+\alpha) \lambda_{p}+\lambda^{0}{ }_{m}+\varphi\left[(1-\alpha) \lambda_{p}-\lambda^{0}{ }_{m}\right]}{(1+\alpha) \lambda_{p}+\lambda^{0}{ }_{m}-\varphi\left[(1-\alpha) \lambda_{p}-\lambda^{0}{ }_{m}\right]}, \\
& \lambda_{\Uparrow}=(1-\varphi) \lambda^{0}{ }_{m}+\varphi \lambda_{p} .
\end{aligned}
$$

$\lambda^{0}{ }_{m}$ is the heat conductivity of the matrix and $\lambda_{p}$ the heat conductivity of the wires. Unlike $\lambda^{0}{ }_{m}$, the quantity $\lambda_{p}$ will incorporate explicitly size effects as made explicit later on in this Section 2. Furthermore, $\varphi$ denotes the volume fraction of the fibers and $\alpha=\frac{r_{K}}{r}$ is a dimensionless parameter taking into account the interface wire-matrix, with $r$ the radius of 
the wire of length $L$ and, $r_{K}$ the so-called Kapitza radius. The latter is expressed by $r_{K}=R \lambda_{m}$, with $R$ designating the interfacial boundary resistance, given by

$$
R=4\left(\frac{1}{c_{m}^{v} v_{m}}+\frac{1}{c_{p}^{v} v_{p}}\right),
$$

where $c^{v_{i}}$ and $v_{i}(i=m, p)$ are the volumetric specific heats and phonon group velocities respectively, note that the result (3) was derived by Chen [13] for pure diffusive scattering. Specularity is taken explicitly into account by introducing a "modified" radius $r^{*}=(1+s) r /(1-$ $s)$ and a "modified" length $L^{*}=(1+s) L /(1-s)$ wherein the parameter $s$ denotes the probability of specular diffusion of the phonons on the particle-matrix interface; $s=0$ is characteristic of diffusive collisions (the quantity $r^{*}$ reduces then to $r$ and $L^{*}$ to $L$ ) while $s=1$ denotes pure specular interactions. In the present section and in the next one, we assume that the temperature is fixed, the effects of a variable temperature will be delayed to section 4 .

The bulk heat conductivity of the host medium is given by the classical expression

$$
\lambda_{m}^{0}=\left.\frac{1}{3} c_{m}^{v} v_{m} \Lambda_{m}\right|_{T r e f}
$$

where $T_{\text {ref }}^{v}$ is the reference temperature, say the room temperature. The mean free path $\Lambda_{m}$ of the phonons inside the matrix is given by the Matthiessen rule:

$$
\frac{1}{\Lambda_{m}}=\frac{1}{\Lambda_{m_{i} b}}+\frac{1}{\Lambda_{m_{i} \text { coll }}}
$$

with $\Lambda_{m, b}$ designating the mean free path in the bulk, $\Lambda_{m, c o n}$ is the supplementary contribution due to the interactions at the particle-matrix interface. Note that although the relation between the mean free path in the matrix and the average separation between 
neighboring nanowires does not appear explicitly in this formulation, it is implicitly included in the expression of $\Lambda_{m, \text { coll }}$. In the case of wires with their axis oriented normally and parallel to the heat flux, this supplementary contribution is given by, respectively, [4,14]:

$$
\frac{1}{\Lambda_{m, \text { coll }}^{\perp}}=\frac{2 \varphi}{r^{*} \pi}, \quad \frac{1}{\Lambda_{m, \text { coll }}^{\| l}}=\varphi\left(\frac{1}{L^{*}}+\frac{2 \zeta}{r^{*} \pi}\right)
$$

wherein $\zeta$ stands for $\zeta=\sqrt{ } \varphi /(\sqrt{ } \varphi+1)$.

To emphasize the role of the size effects on the heat conductivity $\lambda_{p}$ of the nanowires, we will write $\lambda_{p}$ in the form

$$
\lambda_{p}=\lambda_{p}^{0} \lambda_{p}^{S}
$$

wherein $\lambda_{p}{ }^{0}$ contains all the contributions except those linked to the sizes of the nanoparticles which are described by the correcting factor $\lambda_{p}{ }^{S}$. The quantity $\lambda_{p}{ }^{0}$ is given by a relation similar to (4) with sub-index $m$ substituted by $p$ but wherein $\Lambda_{p}$ refers only to the bulk contribution as the effects of ballistic collisions will be included in $\lambda_{p}{ }^{S}$. To determine $\lambda_{p}{ }^{S}$, we will refer to EIT whose main idea is to upgrade the thermodynamic fluxes, like the heat flux and higher order fluxes to the rank of independent variables at the same footing as the energy or the temperature.

For the sake of completeness, let us briefly recall the building up of EIT. The first step consists in assuming that the entropy $\eta\left(e_{,} q\right)$ is not only depending on the internal energy $e$ but besides on the heat flux vector $\boldsymbol{q}$ so that the corresponding Gibbs equation will be written as

$d_{t} \eta\left(e_{p} q\right)=T^{-1} d_{t} e-\gamma_{1} q \cdot d_{t} q$,

wherein $\eta$ and $e$ are measured per unit volume, $T$ is the temperature and $\gamma_{1}$ a 
phenomenological coefficient identified later on. Furthermore, $d_{t}$ denotes the time derivative. However, expression (8) does not account for non-local effects. To introduce such effects, it is appealed to a hierarchy of fluxes $\boldsymbol{Q}^{(1)}, \boldsymbol{Q}^{(2)}, \ldots \boldsymbol{Q}^{(\mathrm{N})}$ with $\boldsymbol{Q}^{(1)}$ identified with the heat flux vector $\boldsymbol{q}, \boldsymbol{Q}^{(2)}$ (a tensor of rank two) is the flux of the heat flux, $\boldsymbol{Q}^{(3)}$ the flux of $\boldsymbol{Q}^{(2)}$, etc. Up to the $\mathrm{n}^{\text {th }}$-order flux, the Gibbs equation generalizing relation (8) becomes $d_{t} \eta\left(e_{n} q_{\nu} Q^{(1)}{ }_{3} \ldots, Q^{(n)}\right)=T^{-1} d_{t} e-\gamma_{1} \boldsymbol{q} \cdot d_{t} q-\gamma_{2} Q^{(2)} \otimes d_{t} Q^{(2)}-\cdots-\gamma_{N} Q^{(n)} \otimes d_{t} Q^{(n)}$,

wherein the symbol $\otimes$ denotes the inner product of the corresponding tensors. The second step is the formulation of the entropy flux $\boldsymbol{J}^{s}$. It is natural to expect that it is not simply given by the classical expression $T^{-1} \boldsymbol{q}$, but that it will depend on higher order fluxes, as

$$
I^{s}=T^{-1} q+\beta_{1} Q^{(2)} \cdot q+\cdots+\beta_{m-1} Q^{(n)} \otimes Q^{(n-1)},
$$

The next step is the derivation of the rate of entropy production per unit volume $\sigma^{\mathrm{s}}$ which is defined by

$$
\sigma^{s}=d_{t} \eta+\nabla . J^{s} \geq 0
$$

and is a positive definite quantity according to the second law of thermodynamics. It is checked that, after substituting in (11) the expressions of $d_{t} \eta$ and $\boldsymbol{J}^{s}$ from (9) and (10) respectively and eliminating $d_{t} e$ via the energy conservation law for rigid heat conductors $\left(d_{t} e=-\nabla \cdot q\right)$, one obtains

$$
\sigma^{s}=-\left(-\nabla T^{-1}+\gamma_{1} d_{t} \boldsymbol{q}-\beta_{1} \nabla \cdot \boldsymbol{Q}^{(2)}\right) \cdot \boldsymbol{q} \ldots-\sum_{n=2}^{N} \boldsymbol{Q}^{(n)} \otimes\left(\gamma_{n} d_{t} \boldsymbol{Q}^{(n)}-\beta_{n} \nabla \cdot \boldsymbol{Q}^{(n+1)}-\beta_{n-1} \nabla Q^{(n-1)}\right) \geq 0
$$

The above bilinear expression in fluxes and forces (the quantities between parentheses) suggests the following hierarchy of linear flux-force relations 


$$
\begin{gathered}
\nabla T^{-1}-\gamma_{1} d_{t} \boldsymbol{q}+\beta_{1} \nabla \cdot \boldsymbol{Q}^{(2)}=\mu_{1} \boldsymbol{q}, \\
\beta_{n-1} \nabla \boldsymbol{Q}^{(n-1)}-\gamma_{n} d_{t} \boldsymbol{Q}^{(n)}+\beta_{n} \nabla \cdot \boldsymbol{Q}^{(n+1)}=\mu_{n} \boldsymbol{Q}^{(n)},(n=2,3 \ldots N .),
\end{gathered}
$$

with $\mu_{1}, \mu_{2}, \ldots \mu_{n}$ being positive phenomenological coefficients to meet the property that $\sigma^{s}$ is positive definite: Equations (13) and (14) can also be seen as time evolution equations for the fluxes $\boldsymbol{q}, \boldsymbol{Q}^{(2)} \ldots Q^{(n)}$. In order to gain insight about the physical meaning of the various phenomenological coefficients, let us assume absence of non-locality so that the term in $\nabla \cdot \boldsymbol{Q}^{(2)}$ will not appear in (13) which reduces to Cattaneo's relation. If in addition, one considers steady situations, the term in $d_{t} \boldsymbol{q}$ vanishes and one recovers Fourier's law. These observations lead to the following identities $\mu_{1}=1 / \lambda T^{2}, \gamma_{1}=\tau / \lambda T^{2}$, indicating that $\mu_{1}$ is related to the heat conductivity $\lambda$ and $\gamma_{1}$ to the relaxation time $\tau$ of the heat flux $\boldsymbol{q}$. The identification of the higher order coefficients demands to compare with higher order evolution equations, but it is expected by analogy that the parameters $\mu_{n}$ and $\gamma_{n}$ are related to coefficients of thermal conductivity $\lambda_{n}$ and relaxation times $\tau_{n}$ of order $n$, respectively. Moreover, since $\boldsymbol{Q}^{(n+1)}$ is the flux of $\boldsymbol{Q}^{(n)}$, this implies, by the very definition of a flux, that $d_{i} Q^{(n)}=-\nabla \cdot Q^{(n+1)}$. Now, when dividing (13) by $\gamma_{1}$ and (14) by $\gamma_{n}(n=2,3, \ldots)$, it follows that $\beta_{1} / \gamma_{1}=-1, \beta_{2} / \gamma_{2}=-1, \ldots$ or, more generally, $\gamma_{\mathrm{n}}=-\beta_{\mathrm{n}}$, which reduces considerably the number of undetermined coefficients.

We assume now that the system is described by an infinite number of flux variables. Applying the spatial Fourier transform $\widehat{\boldsymbol{q}}(\boldsymbol{k}, t)=\int_{-\infty}^{+\infty} \boldsymbol{q}(\boldsymbol{r}, t) e^{-i \boldsymbol{k} \cdot \boldsymbol{r}} d \boldsymbol{r}$ to relations (13) and (14), with $\boldsymbol{k}$ the wave-number vector and $\boldsymbol{r}$ the position vector, one is led to the following Cattaneo-type evolution equation of the Fourier transformed heat flux,

$$
\tau d_{\mathrm{t}} \widehat{q}(k)+\widehat{q}(\boldsymbol{k})=-i k \lambda(k) \widehat{T}(k),
$$


where $\lambda(k)$ is a wavelength-dependent heat conductivity taking the form of a continued fraction expansion [5-7];

$$
\lambda_{p}^{S}(\boldsymbol{k})=\frac{1}{1+\frac{k^{2} l_{1}^{2}}{1+\frac{k^{2} l_{2}^{2}}{1+\frac{k^{2} l_{3}^{2}}{1+\ldots}}},}
$$

where $l_{n}$ are coefficients analogous to the mean free paths associated with the heat flux of order $n$. Suppose for simplicity [15] that all the $l_{n}$ 's are equal $\left(l_{n}=\Lambda_{p} / 2\right)$ with $\Lambda_{p}$ the mean free path of the phonons, by identifying $k$ as $k=2 \pi / \sqrt{\frac{1}{\left(r^{*}\right)^{2}}+\frac{1}{\left(L^{*}\right)^{2}}}$ and defining the Knudsen number $K n$ as $K n=\Lambda_{p} / \sqrt{\frac{1}{\left(r^{*}\right)^{2}}+\frac{1}{\left(L^{*}\right)^{2}}}$, expression (16) has the asymptotic limit [15]

$$
\lambda_{p}^{S}=\frac{1}{2 \pi^{2} K n^{2}}\left(\sqrt{1+4 \pi^{2} K n^{2}}-1\right) .
$$

Note that for sizes of the order of the "de Broglie" wavelength (about $1 \mathrm{~nm}$ for $\mathrm{Si}$, which is the limit size for the validity of Eq. (17)), quantum confinement effects should be taken into account. Note also that Eq. (17) has been used earlier in a different simplified context by Alvarez and Jou [17]. The present work enlarges its domain of applicability by considering a more general class of problems. For small values of $K n$, heat transport is governed by the diffusive regime, i.e. by Fourier's law, and $\lambda_{p}{ }^{s}$ tends to one, which confirms that $\lambda_{p}$ can be identified with its bulk value. For $K n \geq 1$ which is typical of nano configurations with heat transport of ballistic nature, within the limit $K n \rightarrow \infty, \lambda_{p}{ }^{s}$ increases linearly with the radius of the sample in agreement with experimental observations. After combining expressions (7) and (17), one obtains the final expression of the heat conductivity of the nanotubes, namely 


$$
\lambda_{p}=\frac{2}{3} c_{p}^{v} \Lambda_{p} v_{p} \frac{\sqrt{1+4 \pi^{2} K n^{2}}-1}{4 \pi^{2} K n^{2}}
$$

Substitution of (4) and (18) in relations (1) and (2) will allow us to discuss the behavior of the effective thermal conductivity of the nanocomposites in terms of the radial $(r)$ and longitudinal $(L)$ dimensions of the nanowires, their volume fraction $\varphi$, the specularity parameter $s$ and the matrix-particle interface coefficient $\alpha$.

\section{Application to $\mathrm{Si} / \mathrm{Ge}$ nanocomposites}

Let us first consider a Ge host matrix with Si cylindrical nanowire inclusions whose axes are aligned normally to the heat flux. In this Section, the temperature is fixed equal to the room temperature. The values of the bulk parameters used in the calculations are given in Table 1, and are those corresponding to the so-called Debye and dispersive modes $[12,18]$.

Table 1. Numerical values of the parameters at room temperature $\left(T_{r e f}\right)$

$\begin{array}{cccc}\text { Material } & \text { Heat capacity } & \text { Mean free path } l_{b} & \text { Group velocity } \\ & \times 10^{6} \mathrm{~J} / \mathrm{m}^{3} \mathrm{~K} & \mathrm{~nm} & \mathrm{~m} / \mathrm{s}\end{array}$

$\begin{array}{llccc}\mathrm{Si} & \text { Debye } & 1.66 & 40.9 & 6400 \\ & \text { Dispersive } & 0.93 & 260.4 & 1804 \\ \mathrm{Ge} & \text { Debye } & 1.67 & 27.5 & 3900 \\ & & & & 1042\end{array}$

In Fig. 1 is represented the dependence of the effective transversal $\lambda \perp$ heat conductivity of wires as a function of the volume fraction for two values of the radius ( $r=5$ and $r=25 \mathrm{~nm}$ ) and $s=0$ (pure diffusive scattering). Comparison with numerical solutions of the Boltzmann 
transport equation [19] shows an excellent agreement. For a fixed volume fraction, $\lambda \perp$ increases with increasing radii. This is easily understood, as in this case the wire-matrix interface decreases and the phonon interface scattering is less important and offers less resistance against heat transport. Otherwise stated, at larger dimensions, the boundary resistance is weaker and the heat conductivities of both the wires and the matrix become close to their respective bulk values characterized by the absence of boundary effects. When the radius of the wire is fixed, one observes generally a lowering of the thermal conductivity with increasing volume fraction due to the increase of the wire-matrix interface.

In Fig.2 are plotted the values of the transversal heat conductivity versus the volume fraction for two values of the radius $(5$ and $50 \mathrm{~nm})$. The predictions from our model are compared to Fourier's law. In the latter case, the results are not sensitive to size, as it should, and the values of the heat conductivity are systematically overestimated. The effect of the thermal boundary resistance, measured through the parameter $\alpha$ is displayed in Fig. 2 wherein the results of the model are compared with those of a zero Kapitza resistance $\alpha=0$. One notices that the heat conductivity is larger for $\alpha=0$ which is understandable as it corresponds to the weakest boundary resistance. Moreover, the larger is the size of the wire, the greater is the difference between a zero and a non-zero $\alpha$-value, because for larger sizes a change in the value of $\alpha$ will have larger consequences, due to the larger wire-matrix interface. Our results are of the same order of magnitude as those obtained by Behrang et al [14] who limited their analysis to $\alpha=0$. Moreover, their analysis is not based on extended thermodynamics but follows a different route mixing EMA and Boltzmann's theory. Fig. 2 shows that the effect of a non-vanishing $\alpha$ becomes important at large volume fractions and wire sizes. However, since in most nanocomposites, the volume fractions of nanoparticles is relatively small, taking $\alpha \neq 0$ has little impact in actual applications. 


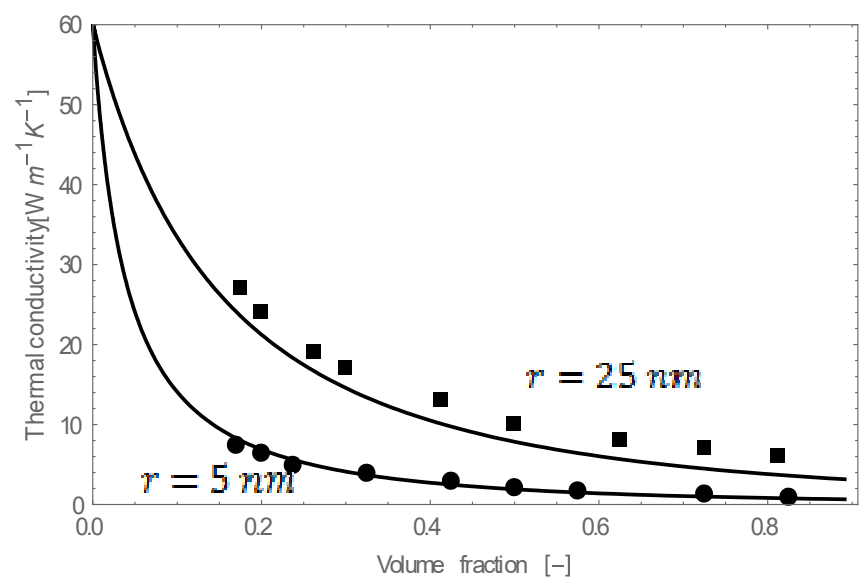

Fig. 1. Transversal effective thermal conductivity of $\mathrm{Si} / \mathrm{Ge}$ nanocomposite as a function of the volume fraction of the nanowires for two values of the radius ( $r=5$ and $25 \mathrm{~nm}$ ) and $s=0$. The results are compared with Boltzmann's solutions represented by circles for $r=5 \mathrm{~nm}$ and squares for $r=25 \mathrm{~nm}$.

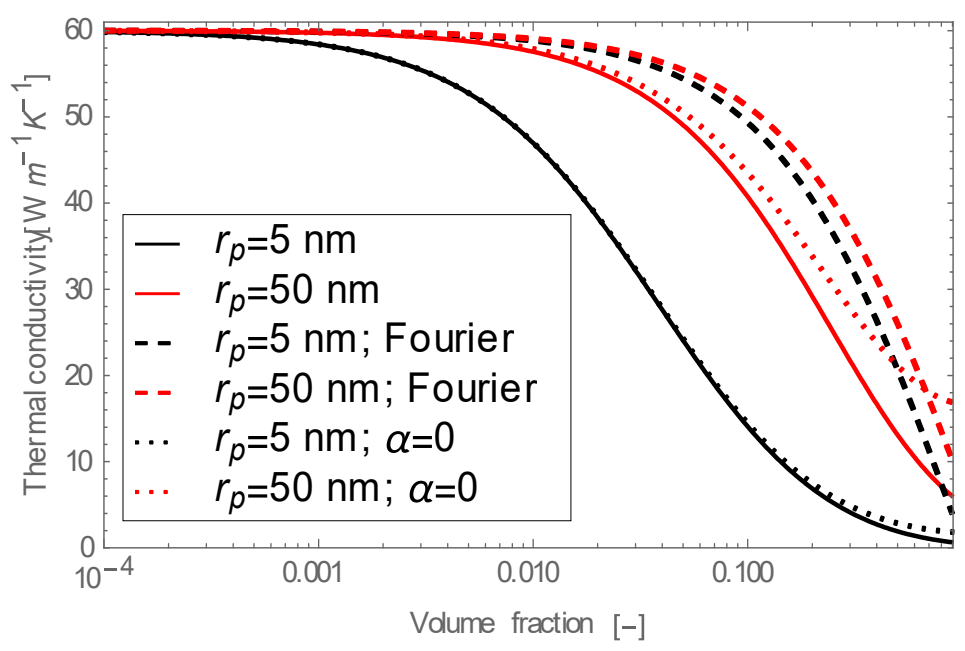

Fig. 2. Transversal effective thermal conductivity versus the volume fraction of nanowires for $r=5,50 \mathrm{~nm}$. Comparison with Fourier's law and with zero Kapitza resistance $\alpha=0$.

In the study of transverse heat conduction, it was admitted that the length of the wires was infinite, in other words much larger than the radii of the wires. To check the validity of 
this approximation, we have calculated the effective heat conductivity $\lambda \perp$ for several lengths of the nanowire at various values of the volume fractions and different sizes. Fig. 3(a) indicates clearly that for a wire radius of $25 \mathrm{~nm}, \lambda \perp$ remains practically constant whatever the length of the wire and the volume fraction. It is true that for large radii, the length has little influences on the transversal heat conductivity. Nevertheless, looking more closely to Fig. 3(b) indicates that for a radius comparable or larger than the length, there is a more significant influence, albeit this effect is much smaller than that of the volume fraction or the wire radius. It is also worth to stress that all the figures have been limited to $\varphi=\pi / \sqrt{ } 12$ which corresponds to the maximum packing of rigid cylindrical nanowires.
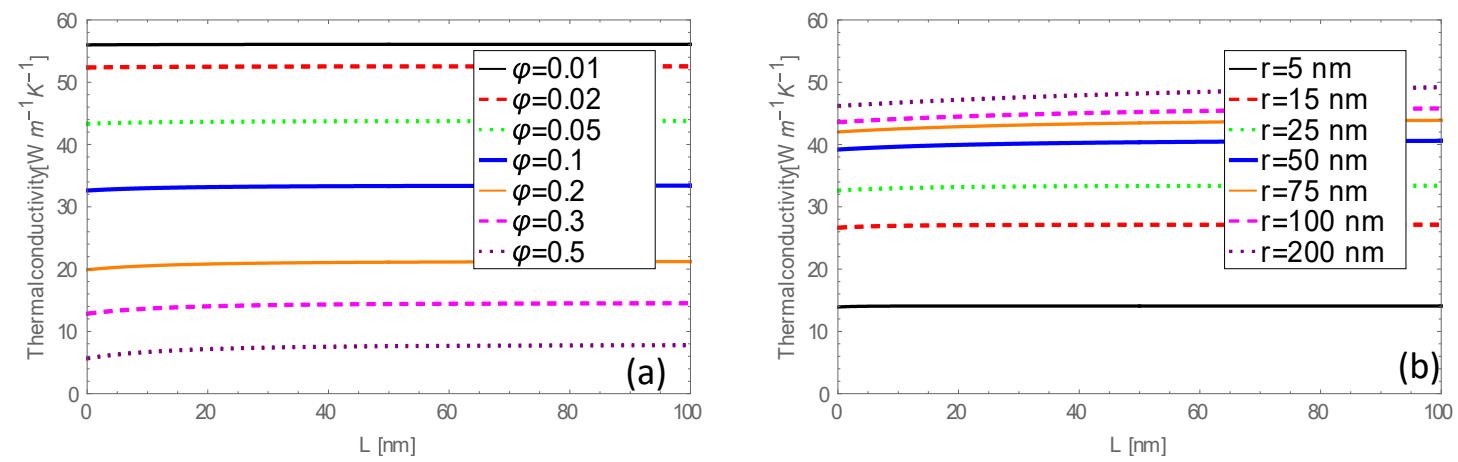

Fig.3. Effect of the longitudinal length $L$ on the transversal heat conductivity for (a) several volume fractions and $r=25 \mathrm{~nm}$ and (b) several wire radii and $\varphi=0.1$.

In the above analysis, it was assumed that the interface was perfect i.e. characterized by $s=0$. In order to appreciate the role of the specularity $s$, we have presented in Fig. 4 the results of the variation of the transversal heat conductivity as a function of the wire length $L$, for several values of $s$. The volume fraction is fixed at $\varphi=0.1$ and three values of the radius are investigated $(r=5,25$ and $100 \mathrm{~nm})$. 


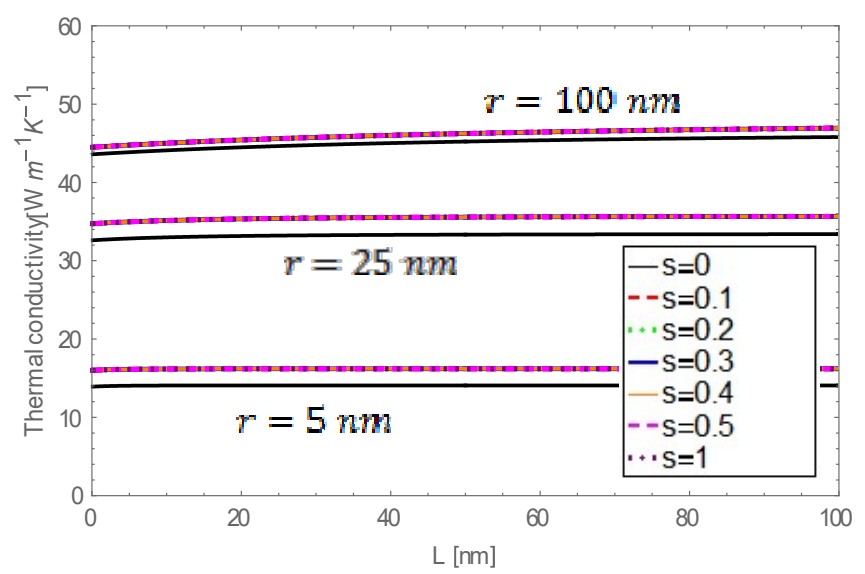

Fig.4. Effect of the longitudinal length $L$ on the transversal heat conductivity for several svalues with $\varphi=0.1$ and $r=5,25,100 \mathrm{~nm}$.

As far as the effect of the $s$-value on the transversal heat conductivity is concerned, we see from Fig. 4 that the only visible differences occur between $s=0$ and $s=0.1$. No significant differences are observed between $s=0.1$ and $s=1$. The main reason is that the flow of phonons normal to the surface is not greatly affected by the nature of the surface; this is no longer true with the longitudinal phonons moving along the interface, as shown below.

The results corresponding to heating in the longitudinal direction are plotted in Fig. 5 for four values of the specularity parameter $(s=0,0.2,0.5$ and 0.9$)$. For $s=0$, heat conductivity is decreasing with the volume fraction and with decreasing sizes. However, by increasing sufficiently either the radius or the s-value (each independently), the heat conductivity increases with the volume fraction. Increasing the values of $s$ means a reduction of the roughness of the particles-matrix interface whence less obstacles are experienced by the phonons and therefore a higher heat conductivity is predicted. Larger wire radii also mean smaller interfaces between the wires and the matrix, with, as a consequence, less obstacles for the phonons and an increase in the heat conductivity. From a mathematical point of view, a larger wire radius and a higher s-value lead to smaller Knudsen numbers, characteristic of the 
Fourier regime. The heat conductivity is then simply a combination of the bulk heat conductivities. Since that of the wires ( $\mathrm{Si})$ is larger than that of the matrix $(\mathrm{Ge})$, the heat conductivity will increase with larger volume fractions. So, whether the longitudinal heat conductivity increases or decreases as a function of the volume fraction depends on the three factors: wire radius, surface specularity and bulk heat conductivities.
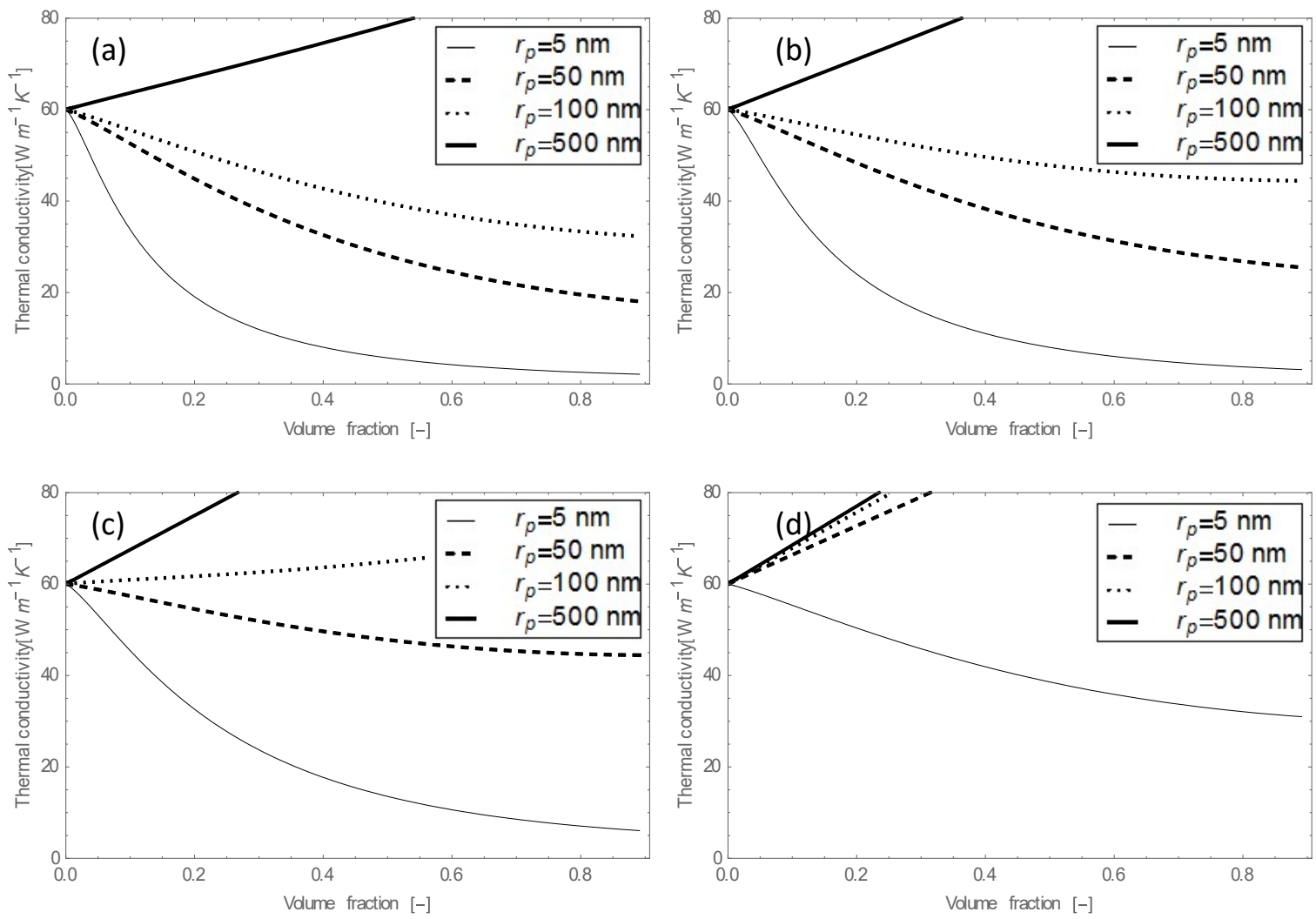

Fig. 5. Longitudinal heat conductivity as a function of volume fraction for four values of the radius $(r=5,50,100,500)$ and various values of $s$ (a) $s=0$, (b) $\mathrm{s}=0.2$, (c) $s=0.5$, (d) $\mathrm{s}=0.9$.

\section{Temperature dependence of the thermal conductivity}

We enlarge the investigations discussed in the previous sections by examining the role of temperature. This is achieved by replacing expression (4) of the heat conductivity by the more general relation wherein the frequency $\omega$ and temperature-dependence are made explicit, namely 


$$
\lambda_{j}=\frac{1}{3} \int_{0}^{\omega_{D}} c_{j}^{v}(\omega, T) v_{j}(\omega, T) l_{j}(\omega, T) d w \quad j=m, p .
$$

The determination of (19) requires the knowledge of $c_{j}^{v}(T, \omega), v_{j}(T, \omega), l_{j, b}(T, \omega)$ for $j=m, p$ and $l_{m, \text { coll }}(T)$ in terms of $\omega$ and $T$. The limit of integration, $\omega_{D}$, is the Debye frequency cutoff: $\omega_{D}=5.1410^{13} \mathrm{~s}^{-1}$ for Ge and $9.1210^{13} \mathrm{~s}^{-1}$ for Si. In agreement with earlier works [20-22], we assume that the phonon group velocity $\boldsymbol{v}$ is independent of the temperature and the frequency. For the specific heat and the mean free path, we take

$$
\begin{aligned}
& c_{j}^{v}=\frac{3 \hbar^{2}}{2 \pi^{2} v_{j}^{3} k_{B} T^{2}} \frac{w^{4} \exp \left(\hbar \omega / k_{B} T\right)}{\left[\exp \left(\hbar \omega / k_{B} T\right)-1\right]^{2}}, j=m, p \\
& \frac{1}{l_{j}}=B_{j} T \omega^{2} \exp \left(-\frac{\theta_{j}}{T}\right), \quad j=m, p
\end{aligned}
$$

wherein $B_{j}$ and $\theta_{j}$ are constant quantities obtained by fitting experimental data: $B_{m}=1.65510^{-}$ ${ }^{22} \mathrm{~s}^{2} \mathrm{~m}^{-1} \mathrm{~K}^{-1}, \theta_{m}=78.92 \mathrm{~K}, B_{p}=5.75310^{-23} \mathrm{~s}^{2} \mathrm{~m}^{-1} \mathrm{~K}^{-1}, \theta_{p}=199.2 \mathrm{~K}$. In the study of temperature dependence, we will use the values of the material data of the Debye model. We have reported in Fig. 6, the dependence of $\lambda_{\text {eff }}$ as a function of temperature for two values of the radius $(r=5$ and $50 \mathrm{~nm})$ and different volume fractions, varying from $\varphi=0.01$ to 0.5 , the wires being oriented normal to the heat flux. The heat conductivity is seen to decrease significantly with the temperature at fixed radius and volume fraction. This behavior can be explained by the fact that the thermal boundary resistance is more important at a lower temperature (lower heat capacities) and therefore contributes to a larger reduction of the heat conductivity. At large volume fraction and small particle size, the heat conductivity is shown to remain almost constant. This is a consequence of the strong particle-matrix interaction that prevails on such conditions. In Fig. 7, the overall thermal conductivities in the longitudinal direction $\lambda_{\|}$are compared with those in the transversal direction $\lambda_{\perp}$ as a function of the 
temperature for several values of $\varphi$ and two different radii. The general behavior is rather similar in the two configurations with the difference that higher values for the thermal conductivity are observed in the longitudinal direction. This is not surprising as phonons experience less boundary scattering while moving in the longitudinal direction.

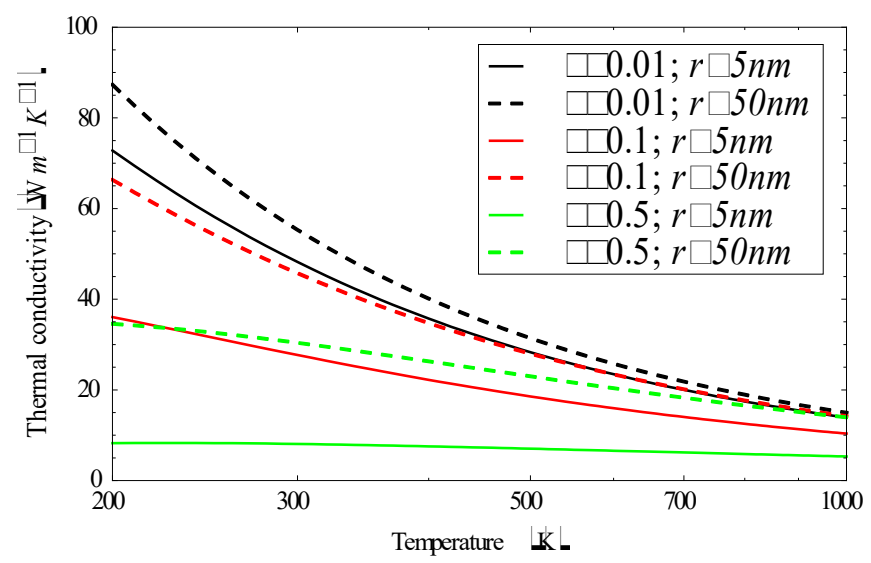

Fig. 6. Thermal dependence of the transversal effective thermal heat conductivity at several volume fractions and two values of the radius: $r=5,50 \mathrm{~nm}$.
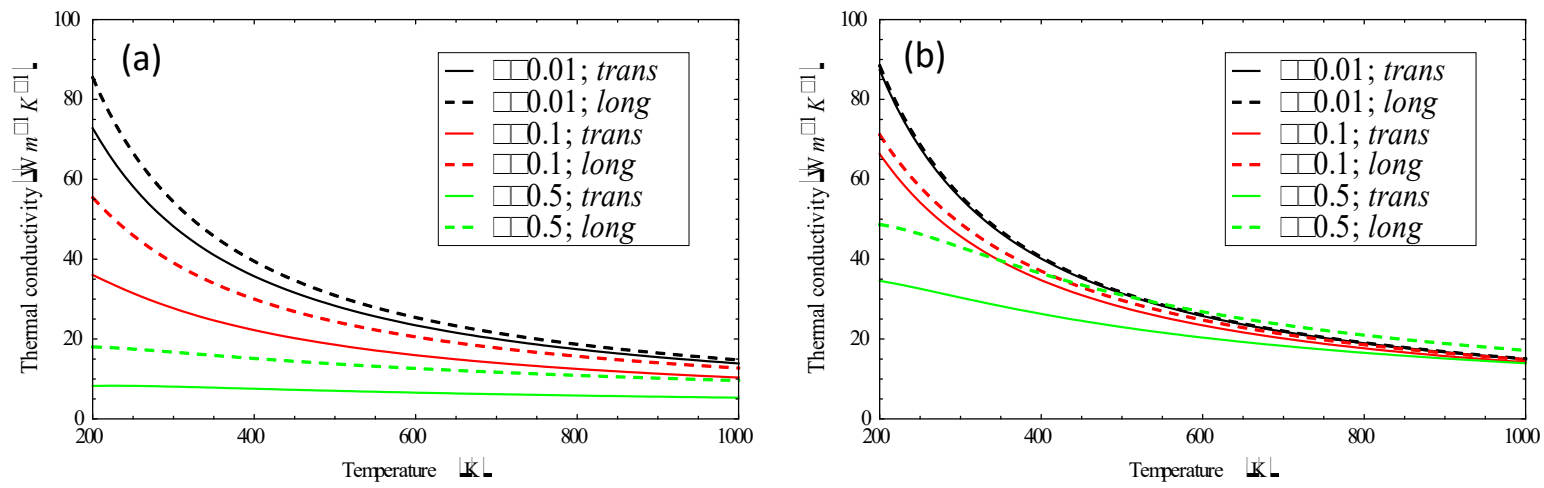

Fig. 7. Comparison between thermal conductivities in the transversal and longitudinal direction respectively as a function of the temperature for different volumes fractions and for $\mathrm{r}=5 \mathrm{~nm}(\mathrm{a})$ and $\mathrm{r}=50 \mathrm{~nm}(\mathrm{~b})$.

\section{Conclusions}


This work is devoted to the study of heat conduction in nanocomposites constituted by dispersed nanowires in a homogeneous matrix. The thermal conductivity depends on several factors as the volume fraction of the nano elements, their size, the nature of the particle-matrix interface and the temperature. Two particular situations have been investigated: nanowires oriented normal and parallel to the heat flow, respectively. The expression of the heat conductivity $\lambda_{m}$ of the matrix is that of the modified EMA model as proposed by Minnich and Chen [4], the main originality of the present approach being the derivation of the expression of the heat conductivity $\lambda_{p}$ of the nanowires. Because their dimensions are comparable to the mean free path of the energy carrier, the classical Fourier law is no longer valid. Instead, we propose for $\lambda_{p}$ a relation derived from Extended Irreversible Thermodynamics [5,6,7], wherein the dependence with respect to the size of the nano wires is made explicit. Special emphasis has been put on the temperature dependence of the heat conductivity, a subject only occasionally treated in the literature. Our results are shown to be in good agreement with those obtained from Boltzmann's transport equation and other approaches following different routes, which attests of the quality of the present modelling. An advantage is that it rests on simple and explicit mathematical expressions which makes it easily tractable from a computing point of view. The above approach was applied to metalloids wherein the phonons are the only heat carriers. In a future work, it is forecast to generalize it by including metallic materials, wherein the heating is governed by both phonons and electrons.

\section{Acknowledgements}

We thank the BelSPo for financial support.

\section{References}

[1] Y. Dong, B.-Y. Cao, Z.-Y. Guo, Physica E 66 (2015) 1. 
[2] C. W. Nan, R. Birringer, D. R. Clarke, H. Gleiter, J. Appl. Phys. 81 (1997).

[3] A.K.M.M. Morshed, T.C. Paul, J.A. Khan, Physica E 47 (2013) 246.

[4] A. J. Minnich, G. Chen, Appl. Phys. Lett. 91 (2007) 073105.

[5] D. Jou, J. Casas-Vazquez, G. Lebon, Extended Irreversible Thermodynamics, 4rth edition, Springer, New York, Dordrecht, Heidelberg, London, 2010.

[6] G. Lebon, D. Jou, J. Casas-Vazquez, Understanding Non-Equilibrium Thermodynamics, Springer, Berlin, 2008.

[7] G. Lebon, J. Non-Equil. Thermodyn. 39 (2014) 35.

[8] H. Machrafi, G. Lebon, Int. J. Nanosc. 13 (2014) 1450022.

[9] A. Behrang, M. Grmela, C. Dubois, S. Turenne, P.G. Lafeur, J. Appl. Phys. 114 (2013) 014305 .

[10] J. Ordonez-Miranda, R. Yang, J.J. Alvarado-Gil, Appl. Phys. Lett. 98 (2011) 233111.

[11] J.C. Maxwell, Treatise on Electricity and Magnetism, 2nd edition, Clarendon, Oxford: 1881.

[12] D.P.H. Hasselman, L.F. Johnson, J. Compos. Mat. 21 (1987) 516.

[13] G. Chen, Phys. Rev. B 57 (1998) 14973.

[14] A. Behrang, M. Grmela, C. Dubois, S. Turenne, P.G. Lafleur, G. Lebon, App. Phys. Lett $.104(2014) 063106$.

[15] S. Hess, Z. Naturforsch. 32a (1977) 678.

[16] D. Jou, J. Casas-Vazquez, G. Lebon, M. Grmela, Appl. Math. Lett. 18 (2005) 963-967.

[17] F. X. Alvarez, D. Jou, Appl. Phys. Lett. 90 (2007) 083109.

[18] G. Chen, Int. J. Therm. Sci. 39 (2000) 471-480.

[19] R. Yang, G. Chen, M.S. Dresselhaus, Phys. Rev. B 72 (2005) 125418.

[20] A. Behrang, M. Grmela, C. Dubois, S. Turenne, P.G. Lafleur, Roy. Soc. Chem. Advances 5 (2015) 2768. 
[21] N. Mingo, L. Yang, D. Li, A. Majumdar, Nano Lett. 3 (2003) 1713-1716.

[22] G. Chen, Nanoscale Energy Transport and Conversion: A Parallel Treatment of Electrons, Molecules, Phonons, and Photons, Oxford University Press, Oxford, 2005. 